\title{
Mitochondrial Organelle Transplantation Is a Potential Therapeutic for Mitochondria Dysfunction in Severe Acute Respiratory Syndrome (SARS) Coronavirus Diseases
}

\author{
Catherine C. Baucom ${ }^{1,2}$, Xian-Peng Jiang2 ${ }^{*}$ (1) \\ ${ }^{1}$ The Mastology Center, Baton Rouge, Louisiana, USA \\ ${ }^{2}$ Sallie Astor Burdine Breast Foundation, Baton Rouge, Louisiana, USA \\ Email: ccbaucom@gmail.com, ‘jiang@breastoncology.com
}

How to cite this paper: Baucom C.C. and Jiang, X.-P. (2021) Mitochondrial Organelle Transplantation Is a Potential Therapeutic for Mitochondria Dysfunction in Severe Acute Respiratory Syndrome (SARS) Coronavirus Diseases. Advances in Infectious Diseases, 11, 298-309.

https://doi.org/10.4236/aid.2021.113027

Received: August 20, 2021

Accepted: September 13, 2021

Published: September 16, 2021

Copyright $\odot 2021$ by author(s) and Scientific Research Publishing Inc. This work is licensed under the Creative Commons Attribution International License (CC BY 4.0).

http://creativecommons.org/licenses/by/4.0/

\begin{abstract}
COVID-19 severe symptoms and high mortality are mainly seen in elders with age-associated diseases who have mitochondrial dysfunction. Mitochondrial dysfunction is a vulnerability and comorbidity of COVID-19. Cytokine storm, and increased serum iron and ferritin and reactive oxygen species (ROS) in COVID-19 further damage mitochondria. Amelioration of mitochondrial dysfunction may be a strategy of prevention and treatment of COVID-19. We also describe mitochondrial organelle transplantation (MOT) which has restored mitochondrial function, improved the repair of injured tissues and suppressed hyperinflammation in life-threatening sepsis. MOT is a potential therapy for severe COVID-19. Finally, we report the first case of MOT for a severe COVID-19 patient. MOT is safe and might have beneficial effect on the severe COVID-19.
\end{abstract}

\section{Keywords}

COVID-19, Mitochondrial Dysfunction, Mitochondrial Organelle

Transplantation, Lung Repair

\section{Introduction}

Severe acute respiratory syndrome coronavirus 2 (SARS-CoV-2) is an enveloped RNA betacoronavirus that causes coronavirus disease 2019 (COVID-19). Up to August 2021, there are over 200 million infectious cases and over 4 million deaths worldwide (https://www.worldometers.info/coronavirus/). Many of the 
patients infected with SARS-CoV-2 are asymptomatic or show low intensity symptoms. However, around $20 \%$ of the patients, mainly elderly people, manifest severe symptoms and high mortality ratio [1] [2]. Severe COVID-19 symptoms are high fever, intense cough, pneumonia, myalgia and acute respiratory distress syndrome (ARDS) [3]. The pathological mechanism of ARDS mainly includes a surge of inflammatory events described as the "cytokine storm". The cytokine storm further causes a series of detrimentally systemic events including oxidative stress, dysregulation of iron homeostasis, hypercoagulability and thrombus formation [4] [5] [6] [7].

Morris G. et al. proposed a model of pathophysiological processes of COVID-19 starting from the infection of human type II alveolar epithelial cells (pneumocytes) by SARS-CoV-2 and culminating in the development of ARDS. The innate immune response to infection of type II alveolar epithelial cells leads both to their death by apoptosis and pyroptosis and to alveolar macrophage activation. Activated macrophages secrete proinflammatory cytokines and chemokines which further activate vascular endothelial cells and recruit highly toxic neutrophils and inflammatory activated platelets into the alveolar space. These activated cells and platelets further produce proinflammatory cytokines and reactive oxygen species (ROS) and contribute to the development of coagulopathy, systemic sepsis, a cytokine storm, ARDS and collapsed lungs [8].

In this review, we will describe that mitochondrial dysfunction is a susceptibility, comorbidity and consequence of COVID-19. Improvement of mitochondrial function may be a strategy of prevention and treatment of COVID-19. We also will discuss an innovative technology, mitochondrial organelle transplantation (MOT), which has been reported to restore mitochondrial function in other disease models. The MOT is a potential therapy for severe COVID-19. Here we will report the first case of MOT therapy for a severe COVID-19 patient. The MOT is safe for the patient who might gain some benefit from the MOT therapy.

\section{Mitochondrial Dysfunction Often Pre-Exists in Patients with Severe COVID-19}

COVID-19 affects essentially elderly people. The age-associated diseases such as metabolic syndrome, obesity, diabetes, and lung and cardiovascular diseases increase the severity and lethality of COVID-19 [1] [9]. Mitochondria are essential for many key activities in cells including ATP energy production, synthesis of nucleotides, synthesis of heme and iron sulfur clusters, iron hemostasis, modification of membrane phospholipids, regulation of calcium homeostasis and innate immunity. Mitochondrial dysfunction is associated with aging and the age-associated diseases. Dysfunctional mitochondria are the main sources of ROS that increase oxidative damage during aging and metabolic diseases [10] [11] [12]. Aging is generally accompanied by a decline in activity of mitochondrial enzymes (e.g. citrate synthase), a decrease in respiratory capacity per mitochondria (e.g. substrate-dependent oxygen consumption), an increase in ROS 
production and a reduced phosphocreatine recovery time (an in vivo measurement of mitochondrial respiratory capacity). Mitochondrial dysfunction can be intimately linked to a wide range of processes associated with aging including senescence, inflammation, as well as the more generalized age-dependent decline in tissue and organ function [13]. Obesity, insulin resistance and type 2 diabetes are accompanied by a variety of systemic and tissue-specific metabolic defects, including inflammation, oxidative and endoplasmic reticulum stress, lipotoxicity and mitochondrial dysfunction [14]. Metabolic syndrome is considered as the co-occurrence of obesity, dyslipidemia and hypertension, main factors for COVID-19 severity [15] [16]. Thus, mitochondrial dysfunction is a key factor for the severity of COVID-19 disease and for triggering the cytokine storm associated with the devastating symptoms that collapse lungs and lead to death to COVID-19 patients. Improvement of mitochondrial activity in the elders would serve as preventive strategy to improve the immune response and reduce mortality caused by COVID-19 and other viral respiratory diseases such as influenza [9].

\section{SARS-CoV-2 May Directly Damage Human Mitochondria}

SARS-CoV-2 is a positive sense RNA coronavirus. SARS-CoV-2 envelope is coated by spike (S) glycoprotein, envelope (E) and membrane (M) proteins. The S1 subunit of the $S$ protein contains the receptor binding domain that binds to the peptidase domain of angiotensin-converting enzyme 2 (ACE 2) [17]. Upon infection, viruses completely rely on host cell molecular machinery to replicate. The viruses target mitochondria and modulate the mitochondrial structure and function [18] [19] [20]. Wu et al. compared hundreds of SARS-CoV-2 genomes with the human transcriptome and other coronaviruses by computational analysis. They predicted that SARS-CoV-2 RNA genome and sgRNAs to be enriched toward the host mitochondrial matrix and nucleolus, and that the 5' and 3' viral untranslated regions contain the strongest, most distinct localization signals. They also interpreted the mitochondrial residency signal as an indicator of intracellular RNA trafficking with respect to double-membrane vesicles, a critical stage in the coronavirus life cycle [21]. However, it has been not yet known whether noncoding RNAs of coronavirus inhibit mitochondrial transcription, protein translation, or tRNA processing. Pasquier $\mathrm{C}$ et al. reported that ORF3a includes a 20-base sequence that targets USP30, a mitochondrial ubiquitin-specific peptidase 30. The 20 nucleotides present in ORF3a of SARS-CoV-2 target the sequence AAAGATAGAGAAAAGGGGCT found in USP30 transcripts. USP30 is a mitochondrial deubiquitinase involved in mitochondria homeostasis and controls mitophagy. SARS-CoV-2 might affect mitochondria function by altering ubiquitination and contribute to the suppression of immunity in COVID-19 patients [22].

SARS-CoV protein can localize into host mitochondria. SARS-CoV-1 virus upon infection affects mitochondrial functions, influences its intracellular sur- 
vival, or evades host immunity. SARS-CoV-1 ORF-9b targets into host mitochondria, which suppresses innate immunity by manipulating mitochondrial function and mitochondrial antiviral signaling protein (MAVS)/TNF receptor-associated factor (TRAF)3/TRAF6 signaling pathway to host innate immunity [23]. Furthermore, SARS-COV-1 ORF3b [24], ORF7a [25], and ORF8a also localize to mitochondria and promote viral replication [26]. With the exception of ORF3b, SARS-CoV-2 encodes amino acid sequences are very similar to SARS-CoV-1 ORFs (ORF7a, -8a, and -9b) proven to be localized to host mitochondria [20]. Many proteins of SARS-CoV-2 interact with host mitochondrial proteins, for example, Nsp8 interaction with mitochondrial MRPS2, MRPS5, MRPS25, and MRPS27 ribosomal proteins, ORF9c interaction with mitochondrial NDUFAF1 and NDUFB9, and Nsp7 interaction with mitochondrial NDUFAF2. Notably, both NDUFAF1 and 2 play a critical role in the assembly of complex I. NDUFB9 is an essential subunit of complex I comprised of more than 40 subunits. Furthermore, viral $M$ protein was described to interact with ATP1B1, ATP6V1A, ACADM, AASS, PMPCB, PITRM1, COQ8B, and PMPCA. These proteins are part of the critical metabolic pathways carrying mitochondrial metabolism [27]. The effects of protein-protein interaction on mitochondrial function remain to be determined by experiments.

\section{The Pathology of COVID-19 Leads to the Mitochondrial Dysfunction}

\subsection{ROS Accumulation in COVID-19}

Mitochondria are the major source of ROS that contributes to normal cell function, but also increases intracellular oxidative stress. The peroxisome proliferator-activated receptor-gamma coactivator (PGC)-1alpha plays an important key role in balancing mitochondrial biogenesis and antioxidation that protect cells from ROS oxidative damage [28] [29]. During inflammation, low levels of PGC-1 $\alpha$ downregulate mitochondrial antioxidant gene expression, induce oxidative stress, and promote nuclear factor kappa B activation. In aging and metabolic dysfunction such as obesity, Type 2 diabetes mellitus, hepatic steatosis and cardiovascular disease, the levels of PGC-1a in organs are decreased. PGC-1a downregulation modifies the metabolic properties of tissues by altering mitochondrial function and promoting ROS accumulation. Thus, the mitochondria in aging and metabolic dysfunction are vulnerable to ROS and other harmful factors [29]. In cytokine storm of severe COVID-19, cytokines such as TNF-a, INF-r, IL-6, IL-10, etc. cause an increase in mitochondrial ROS production, impair mitochondrial oxidative phosphorylation and modulate the electron transport chain. Those also cause mitochondrial permeabilization, alter mitochondrial dynamics and ultimately result in apoptosis [6]. Mitochondrial ROS directly stimulate the production of proinflammatory cytokines [30]. The vicious inflammatory/oxidative cycles lead to further mitochondrial injury and acute lung damage in severe COVID-19. 


\subsection{Elevated Serum Iron and Ferritin}

In severe COVID-19, elevated systemic iron and ferritin cause the increased free radicals and ROS, disrupt mitochondrial homeostasis, induce ferroptosis, and drive mitochondrial respiration from aerobic into anaerobic state [6] [31].

\subsection{Damage of Extracellular Mitochondria}

Extracellular mitochondria can be found as free and enclosed by a membrane inside platelets or vesicles. Mitochondria outside cells can induce paracrine or endocrine responses in an organism. Extracellular mitochondria regulate cell-to-cell communication, regeneration and danger sensing and provoke an immune response [32] [33]. Each platelet packages 5 - 8 mitochondria which are critical for vital platelet functions including aerobic respiration and metabolism [34]. Thrombocytopenia and hypercoagulability are associated with COVID-19 severity mostly in non-survivors [35]. The increased platelet damage inevitably leads to the diminishment and abnormality of extracellular mitochondria.

\subsection{Mitochondrial Dysfunction in Peripheral Blood Mononuclear Cells (PBMC)}

Clinical research has shown the mitochondrial dysfunction in periphery blood cells [36] [37]. Ajaz S. et al. reported PBMC of COVID-19 patients showed significant decreases in basal respiration, ATP-linked respiration, reserve capacity and maximal respiration, and no change of nonmitochondrial respiration and proton leak, compared to health individuals and patients with chest infection. The glycolysis is increased in PBMC of COVID-19 patients [36]. Gibellini L. et al. examined PBMC from patients with COVID-19 pneumonia and found that these cells had impaired bioenergetics and mitochondrial dysfunction with a reduced basal and maximal respiration, reduced spare respiratory capacity and decreased proton leak. Basal extracellular acidification rate was also reduced, suggesting reduced capability to perform aerobic glycolysis. Although COVID-19 monocytes had a reduced ability to perform oxidative burst, they were still capable of producing TNF and IFN- $\gamma$ in vitro. Transmission electron microscopy showed abnormal mitochondrial ultrastructure in monocytes [37].

\section{MOT May Restore Mitochondrial Function and Improve Lung Regeneration in COVID-19 Patients}

In COVID-19, both intracellular and extracellular mitochondria are impaired. Therefore, replenishment of healthy mitochondria is a potential therapy for severe COVID-19.

\subsection{Mitochondrial Transfer between Cells}

Mitochondria can transfer between cells. Mesenchymal stem cells (MSCs) can refill the healthy mitochondria to the injured cells [38] [39] [40] [41]. In 2006, Spees et al. reported that mitochondria of stem cells transferred to the mito- 
chondrial DNA (mtDNA)-depleted human epithelial cells A549. The mitochondrial DNA and oxidative phosphorylation were recovered after co-culture of stem cells and the mtDNA-depleted A549 [38]. Paliwal S. et al. reviewed repair and regeneration of damaged cells or tissues by MSCs. They have discussed the phenomenon of mitochondrial transfer from MSCs to neighboring stressed cells, and it aids cellular repair and regeneration of different organs such as lung, heart, eye, brain and kidney. Mitochondrial transfer appears to be a gainful strategy for regenerative processes, as mitochondrial biosynthesis usually takes longer than that can be afforded by damaged cells in crisis. Thus, mitochondrial transfer is an efficient method for regeneration of injured cells. Various mechanisms are involved in mitochondrial transfer that includes tunnel tube formation, micro vesicle formation, gap junctions, cell fusion and other modes of transfer. Remarkable restoration of cellular bioenergetics and reduction in oxidative stress in all the studies of MSCs that demonstrates that mitochondrial transfer from MSCs plays a critical role in cellular repair and regeneration [39]. Islam et al. reported that mitochondrial transfer from bone-marrow-derived stromal cells to pulmonary alveoli protects against acute lung injury [40]. In other study, MSCs rescue injured endothelial cells in an in vitro ischemia-reperfusion model via tunneling nanotube like structure-mediated mitochondrial transfer [41]. These studies suggest mitochondrial transfer as one of prominent mechanisms through which MSCs can regenerate and repair damaged cells or tissues. MSCs lack angiotensin-converting enzyme-2 (ACE2) receptor, which is a receptor widely distributed on the surface of human cells and required for the entry of coronavirus into host cells. Such property ensures that injected MSCs can accomplish immunomodulatory effects without being destroyed by the virus. MSCs therapy has been reported to ease the cytokine storm syndrome and significantly improve the repair of damaged tissues and the outcome in severe COVID-19 patients [42] [43] [44]. However, the underlying mechanism of mitochondrial transfer from MSCs to injured cells in COVID patients appears to be neglected.

\subsection{MOT with Isolated Mitochondria}

MOT is an innovative technology to transfer the isolated healthy mitochondria to injured or diseased cells. We published that the mitochondria isolated from primary fibroblasts actively transplanted to the mitochondrial DNA (mtDNA)-depleted mouse motor neuron NSC-34 cells (NSC-34 $\left.\rho^{0}\right)$ after co-culture, and the mitochondrial transplantation replenished mtDNA (i.e., genes of mitochondrially encoded cytochrome $c$ oxidase I and mitochondrially encoded NADH dehydrogenase 1) and rescued aerobic respiration of NSC-34 $\rho^{0}$ cells [45]. Early our research showed that human normal mammary epithelial mitochondria transplanted into human breast cancer cell line MCF-7, T47D and MDA-MB-231, and suppressed glycolytic metabolism and glucose uptake of human breast cancer cells and reduced lactate production [46]. MOT has been 
studied in animal models. Masuzawa et al. reported that transplantation of autologously derived mitochondria could protect the heart from ischemia reperfusion injury [47]. The mitochondria were obtained from small biopsies from the pectoralis major muscle of New Zealand whited rabbits. The isolated autologous mitochondria injected into the hearts were in the interstitial spaces surrounding cardiomyocytes at $0,2,4,8$, and 24 hours after injection. There was an extensive subendocardial distribution and labeled mitochondria were localized within cardiomyocytes at 2 hours after injection. These internalized mitochondria maintained viability and function producing adequate ATP levels. Importantly, the transplanted mitochondria decreased inflammatory markers and did not promote autoimmunity or arrhythmia. Their studies have also demonstrated that once transplanted, exogenous mitochondria provided cardioprotection both extracellularly and intracellularly. Non-viable mitochondria, mitochondrial fractions, mtDNA or RNA, and exogenous ATP or ADP did not confer cardioprotection when administered into the ischemic heart [47] [48]. Moskowitzova et al. recently published that mitochondrial transplantation by vascular delivery or nebulization improved lung mechanics, decreased lung tissue injury, and enhanced murine lung viability and recovery after ischemia-reperfusion injury [49].

\subsection{Exogenous Mitochondria Have Immunomodulatory Effect}

MOT also shows immunomodulatory effects. Hwang et al. reported that intravenously mitochondrial administration increased mitochondrial respiration in spleen and muscle tissue in the cecal slurry rat model. MOT significantly reduced the bacterial burden in both spleen and blood. The lactate level was decreased by mitochondrial injection. MOT had an immunosuppressive effect in the hyperinflammatory phase, as shown by reduced TNF- $\alpha$ levels in the spleen, and an immune-enhancing effect in the late immune paralysis phase of sepsis. These results implied that mitochondrial transplantation had a bimodal immunomodulatory effect on sepsis. The survival rate significantly increased with MOT from $10 \%$ to $50 \%$. MOT could be a promising new therapeutic approach for life-threatening sepsis cases [50].

In severe COVID-19, mitochondrial dysfunction, cytokine storm and vicious inflammatory/oxidative cycles lead to acute lung damage and multiple organ failure. MOT could not only restore the mitochondrial function, replenish extracellular mitochondria and improves the repair of cells and tissues, but also act as an immunomodulation in SARS-CoV-2 induced sepsis. Due to mitochondrial dysfunction is early event of COVID-19, the earlier MOT in COVID elder patients with the age-associated diseases could achieve the better beneficial effects.

\section{Case Report: MOT in a Severe COVID-19 Patient}

An 84-year-old healthy man who had devoted the last 15 years of his life to stud- 
ying mitochondrial function, contracted COVID-19. His only pre-existing medical condition was hypertension. His symptoms were initially mild with low grade fever and runny nose. Over the next 14 days, his symptoms progressed to pneumonia and his oxygen saturation at the time of presentation to the emergency room was $70 \%$. Over the next several weeks, his oxygen requirements continued to escalate. There was multiple pneumothorax. Several different oxygenation methods were used; however, ultimately he required intubation and became ventilator dependent.

There was no clinical treatment option for the patient. Under compassionate care and with the family approval, the hospital approved the MOT with the waiver of Institute Review Board (IRB). $5 \mathrm{ml}$ of mitochondrial solution were administered intravenously. This intravenous solution contained about the mitochondria isolated from 50 million primary human fibroblasts. The patient was observed for about 1 hour following administration of the mitochondria without any adverse effects observed. Over the next several days, the patient's oxygenation requirement was improved, and his oxygenation was weaned down on the ventilator. He progressed to having spontaneous breathing trials. Unfortunately, his recovery was complicated by a large pneumothorax which required several chest tubes. A week later, the patient received another $5 \mathrm{ml}$ injection of mitochondria. No adverse effects were observed. The clinical response to the 2nd MOT treatment was diminished due to the progression of COVID-19 lung damage progressing into pulmonary fibrosis. With a poor prognosis, the family elected to withdraw care and the patient passed away from complications of COVID-19.

In this clinical case, the first MOT intravenous injection showed improvement in pulmonary function based on improved ventilator settings and decrease in oxygenation requirements. MOT had no adverse effect. Treatment of cytokine storm from COVID-19 infections including steroids and antibiotics can adversely affect mitochondria. Early treatment with MOT prior to progression of the cytokine storm may show a greater benefit in recovery from severe COVID-19 infections.

\section{Summary and Conclusion}

SARS-CoV-2 causes severe COVID-19 and high mortality mainly in the group of elder and age-associated diseases who have dysfunctional mitochondria. SARS-CoV-2 itself and COVID pathologies could further damage mitochondria. These result in acute severe metabolic abnormalities which lead to multiple organ failure, death, or long-lasting metabolic-health complications in survivors of severe COVID-19. Support of optimal mitochondrial function would serve as prevention to improve the immune response and treatment to reduce mortality caused by COVID-19. MOT has been shown to replenish mitochondrial function in the diseased cells and animal models and improve the repair and regeneration of the injured cells and tissues. Exogenous healthy mitochondria also act 
as an immunomodulation in sepsis. Therefore, MOT might be a cellular therapy for severe COVID-19. We first gave MOT to a severe COVID-19. The MOT was safe and might have some efficacy in the severe COVID-19 patient.

\section{Acknowledgements}

The study was supported by the Sallie Astor Burdine Breast Foundation, Baton Rouge, Louisiana, USA.

\section{Data Availability}

The datasets used and/or analyzed during the current study are available from the first author on reasonable request.

\section{Conflicts of Interest}

The authors declare no conflicts of interest regarding the publication of this paper.

\section{References}

[1] Chen, J., Kelley, W.J. and Goldstein, D.R. (2020) Role of Aging and the Immune Response to Respiratory Viral Infections: Potential Implications for COVID-19. The Journal of Immunology, 205, 313-320. https://doi.org/10.4049/jimmunol.2000380

[2] Onder, G., Rezza, G. and Silvio Brusaferro, S. (2020) Case-Fatality Rate and Characteristics of Patients Dying in Relation to COVID-19 in Italy. JAMA, 323, 1775-1776. https://doi.org/10.1001/jama.2020.4683

[3] Rothan, H.A. and Byrareddy, S.N. (2020) The Epidemiology and Pathogenesis of Coronavirus Disease (COVID-19) Outbreak. Journal of Autoimmunity, 109, Article ID: 102433. https://doi.org/10.1016/j.jaut.2020.102433

[4] Moore, B.J.B. and June, C.H. (2020) Cytokine Release Syndrome in Severe COVID-19. Science, 368, 473-474. https://doi.org/10.1126/science.abb8925

[5] Phua, J., Weng, L., Ling, L., Egi, M., Lim, C.M., Divatia, J.V., et al. (2020) Intensive Care Management of Coronavirus Disease 2019 (COVID-19): Challenges and Recommendations. The Lancet Respiratory Medicine, 8, 506-517. https://doi.org/10.1016/S2213-2600(20)30161-2

[6] Saleha, J., Peyssonnaux, C., Singh, K.K. and Edeas, M. (2020) Mitochondria and Microbiota Dysfunction in COVID-19 Pathogenesis. Mitochondrion, 54, 1-7. https://doi.org/10.1016/j.mito.2020.06.008

[7] Zhou, F., Yu, T., Du, R., Fan, G., Liu, Y., Liu, Z., et al. (2020) Clinical Course and Risk Factors for Mortality of Adult Inpatients with COVID-19 in Wuhan, China: A Retrospective Cohort Study. The Lancet, 395, 1054-1062. https://doi.org/10.1016/S0140-6736(20)30566-3

[8] Morris, G., Bortolasci, C.C., Puri, B.K., Olive, L., Marx, W., O’Neil, A., et al. (2020) The Pathophysiology of SARS-CoV-2: A Suggested Model and Therapeutic Approach. Life Sciences, 258, Article ID: 118166. https://doi.org/10.1016/j.lfs.2020.118166

[9] Fernández-Ayala, D.J.M., Navas, P. and López-Lluch, G. (2020) Age-Related Mitochondrial Dysfunction as a Key Factor in COVID-19 Disease. Experimental Gerontology, 142, Article ID: 111147. https://doi.org/10.1016/j.exger.2020.111147 
[10] Horowitz, M.P. and Greenamyre, J.T. (2010) Mitochondrial Iron Metabolism and Its Role in Neurodegeneration. Journal of Alzheimer's Disease, 20, S551-S568. https://doi.org/10.3233/JAD-2010-100354

[11] Weinberg, S.E., Sena, L.A. and Chandel, N.S. (2015) Mitochondria in the Regulation of Innate and Adaptive Immunity. Immunity, 42, 406-417.

https://doi.org/10.1016/j.immuni.2015.02.002

[12] Lopez-Lluch, G. (2018) Mitochondrial Dysfunction in Metabolism and Ageing: Shared Mechanisms and Outcomes? Biogerontology, 19, 461-480.

https://doi.org/10.1007/s10522-018-9768-2

[13] Haas, R.H. (2019) Mitochondrial Dysfunction in Aging and Diseases of Aging. Bi$\operatorname{ology}$ (Basel), 8, 48. https://doi.org/10.3390/biology8020048

[14] Kim, J.A., Wei, Y. and Sowers, J.R. (2008) Role of Mitochondrial Dysfunction in Insulin Resistance. Circulation Research, 102, 401-414.

https://doi.org/10.1161/CIRCRESAHA.107.165472

[15] Prasun, P. (2020) Mitochondrial Dysfunction in Metabolic Syndrome. Biochimica et Biophysica Acta (BBA)-Molecular Basis of Disease, 1866, Article ID: 165838. https://doi.org/10.1016/j.bbadis.2020.165838

[16] Zaki, N., Alashwal, H. and Ibrahim, S. (2020) Association of Hypertension, Diabetes, Stroke, Cancer, Kidney Disease, and High-Cholesterol with COVID-19 Disease Severity and Fatality: A Systematic Review. Diabetology \& Metabolic Syndrome, 14, 1133-1142. https://doi.org/10.1016/j.dsx.2020.07.005

[17] Cevik, M., Kuppalli, K., Kindrachuk, J. and Peiris, M. (2020) Virology, Transmission, and Pathogenesis of SARS-CoV-2. BMJ, 371, m3862.

https://doi.org/10.1136/bmj.m3862

[18] Burtscher, J., Cappellano, G., Omori, A., Koshiba, T. and Millet, G.P. (2020) Mitochondria: In the Cross Fire of SARS-CoV-2 and Immunity. iScience, 23, Article ID: 101631. https://doi.org/10.1016/j.isci.2020.101631

[19] Gatti, P., Ilamathi, H.S., Todkar, K. and Germain, M. (2020) Mitochondria Targeted Viral Replication and Survival Strategies-Prospective on SARS-CoV-2. Frontiers in Pharmacology, 11, Article ID: 578599. https://doi.org/10.3389/fphar.2020.578599

[20] Singh, K.K., Chaubey, G., Chen, J.Y. and Suravajhala, P. (2020) Decoding SARS-CoV-2 Hijacking of Host Mitochondria in COVID-19 Pathogenesis. American Journal of Physiology-Cell Physiology, 319, C258-C267. https://doi.org/10.1152/ajpcell.00224.2020

[21] Wu, K.E., Fazal, F.M., Parker, K.R., Zou, J. and Chang, H.Y. (2020) RNA-GPS Predicts SARS-CoV-2 RNA Residency to Host Mitochondria and Nucleolus. Cell Systems, 11, 102-108. https://doi.org/10.1016/j.cels.2020.06.008

[22] Pasquier, C. and Robichon, A. (2020) SARS-CoV-2 Might Manipulate against Its Host the Immunity RNAi/Dicer/Ago System. Does Mitochondria Collapse upon COVID-10 Infection?

[23] Shi, C.S., Qi, H.Y., Boularan, C., Huang, N.N., Abu-Asab, M., Shelhamer, J.H. and Kehrl, J.H. (2014) SARS-Coronavirus Open Reading Frame-9b Suppresses Innate Immunity by Targeting Mitochondria and the MAVS/TRAF3/TRAF6 Signalosome. The Journal of Immunology, 193, 3080-3089. https://doi.org/10.4049/jimmunol.1303196

[24] Freundt, E.C., Yu, L., Park, E., Lenardo, M.J. and Xu, X.N. (2009) Molecular Determinants for Subcellular Localization of the Severe Acute Respiratory Syndrome Coronavirus Open Reading Frame 3b Protein. Journal of Virology, 83, 6631-6640. https://doi.org/10.1128/JVI.00367-09 
[25] Tan, Y.X., Tan, T.H., Lee, M.J.R., Tham, P.Y., Gunalan, V., Druce, J., et al. (2007) Induction of Apoptosis by the Severe Acute Respiratory Syndrome Coronavirus 7a Protein Is Dependent on Its Interaction with the Bcl-XL Protein. Journal of Virology, 81, 6346-6355. https://doi.org/10.1128/JVI.00090-07

[26] Chen, C.Y., Ping, Y.H., Lee, H.C., Chen, K.H., Lee, Y.M., Chan, Y.J., et al. (2007) Open Reading Frame 8a of the Human Severe Acute Respiratory Syndrome Coronavirus Not Only Promotes Viral Replication But Also Induces Apoptosis. The Journal of Infectious Diseases, 196, 405-415. https://doi.org/10.1086/519166

[27] Gordon, D.E., Jang, G.M., Bouhaddou, M., Xu, J., Obernier, K., O’Meara, M.J., et al. (2020) A SARS-CoV-2-Human Protein-Protein Interaction Map Reveals Drug Targets and Potential Drug-Repurposing. https://doi.org/10.1101/2020.03.22.002386

[28] Liang, H.Y. and Ward, W.F. (2006) PGC-1alpha: A Key Regulator of Energy Metabolism. Advances in Physiology Education, 30, 145-151.

https://doi.org/10.1152/advan.00052.2006

[29] Rius-Pérez, S., Torres-Cuevas, I., Millán, I., Ortega, A.L. and Pérez, S. (2020) PGC-1 $\alpha$, Inflammation, and Oxidative Stress: An Integrative View in Metabolism. Oxidative Medicine and Cellular Longevity, 2020, Article ID: 1452696. https://doi.org/10.1155/2020/1452696

[30] Li, X., Fang, P., Mai, J., Choi, E.T., Wang, H. and Yang, X.F. (2013) Targeting Mitochondrial Reactive Oxygen Species as Novel Therapy for Inflammatory Diseases and Cancers. Journal of Hematology \& Oncology, 6, 1-19. https://doi.org/10.1186/1756-8722-6-19

[31] Huang, C., Wang, Y., Li, X., Ren, L., Zhao, J., Zhang, L., et al. (2020) Clinical Features of Patients Infected with 2019 Novel Coronavirus in Wuhan, China. The Lancet, 395, 497-506. https://doi.org/10.1016/S0140-6736(20)30183-5

[32] Wang, L., Wu, Q., Fan, Z., Xie, R., Wang, Z. and Lu, Y. (2017) Platelet Mitochondrial Dysfunction and the Correlation with Human Diseases. Biochemical Society Transactions, 45, 1213-1223. https://doi.org/10.1042/BST20170291

[33] Miliotis, S., Nicolalde, B., Ortega, M., Yepez, J. and Caicedo, A. (2019) Forms of Extracellular Mitochondria and Their Impact in Health. Mitochondrion, 48, 16-30. https://doi.org/10.1016/j.mito.2019.02.002

[34] Melchinger, H., Jain, K., Tyagi, T. and Hwa, J. (2019) Role of Platelet Mitochondria: Life in a Nucleus-Free Zone. Frontiers in Cardiovascular Medicine, 6, 1-11. https://doi.org/10.3389/fcvm.2019.00153

[35] Lippi, G., Plebani, M. and Henry, B.M. (2020) Thrombocytopenia Is Associated with Severe Coronavirus Disease 2019 (COVID-19) Infections: A Meta-Analysis. Clinica Chimica Acta, 506, 145-148. https://doi.org/10.1016/j.cca.2020.03.022

[36] Ajaz, S., McPhail, M.J., Singh, K.K., Mujib, S., Trovato, F.M., Napoli, S. and Agarwal, K. (2020) Mitochondrial Metabolic Manipulation by SARS-CoV-2 in Peripheral Blood Mononuclear Cells of Patients with COVID-19. American Journal of Physiology-Cell Physiology, 320, C57-C65. https://doi.org/10.1152/ajpcell.00426.2020

[37] Gibellini, L., Biasi, S.D., Paolini, A., Borella, R., Boraldi, F., Mattioli, M., et al. (2020) Altered Bioenergetics and Mitochondrial Dysfunction of Monocytes in Patients with COVID-19 Pneumonia. EMBO Molecular Medicine, 12, e13001. https://doi.org/10.15252/emmm.202013001

[38] Spees, J.L., Olson, S.D., Whitney, M.J. and Prockop, D.J. (2006) Mitochondrial Transfer between Cells Can Rescue Aerobic Respiration. Proceedings of the National Academy of Sciences of the United States of America, 103, 1283-1288. https://doi.org/10.1073/pnas.0510511103 
[39] Paliwal, S., Chaudhuri, R., Agrawal, A. and Mohanty, S. (2018) Regenerative Abilities of Mesenchymal Stem Cells through Mitochondrial Transfer. Journal of Biomedical Science, 25, 31. https://doi.org/10.1186/s12929-018-0429-1

[40] Islam, M.N., Das, S.R., Emin, M.T., Wei, M., Sun, L., Westphalen, K., et al. (2012) Mitochondrial Transfer from Bone Marrow-Derived Stromal Cells to Pulmonary Alveoli Protects against Acute Lung Injury. Nature Medicine, 18, 759-765. https://doi.org/10.1038/nm.2736

[41] Liu, K., Ji, K., Guo, L., Wu, W., Lu, H., Shan, P. and Yan, C. (2014) Mesenchymal Stem Cells Rescue Injured Endothelial Cells in an in Vitro Ischemia-Reperfusion Model via Tunneling Nanotube Like Structure-Mediated Mitochondrial Transfer. Microvascular Research, 92, 10-18. https://doi.org/10.1016/j.mvr.2014.01.008

[42] Leng, Z., Zhu, R., Hou, W., Feng, Y., Yang, Y., Han, Q., et al. (2020) Transplantation of ACE2-Mesenchymal Stem Cells Improves the Outcome of Patients with COVID-19 Pneumonia. Aging and Disease, 11, 216-228. https://doi.org/10.14336/AD.2020.0228

[43] Rajarshi, K., Chatterjee, A. and Shashikant Ray, S. (2020) Combating COVID-19 with Mesenchymal Stem Cell Therapy. Biotechnology Reports, 26, e00467. https://doi.org/10.1016/j.btre.2020.e00467

[44] Wang, S.H., Shetty, A.K., Jin, K. and Zhao, R.C. (2020) Combating COVID-19 with Mesenchymal Stem/Stromal Cell Therapy: Promise and Challenges. Frontiers in Cell and Developmental Biology, 8, Article ID: 627414. https://doi.org/10.3389/fcell.2020.627414

[45] Jiang, X.P., Baucom, C.C. and Elliott, R.L. (2020) Mitochondria Dynamically Transplant into Cells in Vitro and in Mice and Rescue Aerobic Respiration of Mitochondrial DNA-Depleted Motor Neuron NSC-34. Journal of Biomedical Science and Engineering, 13, 203-221. https://doi.org/10.4236/jbise.2020.139019

[46] Jiang, X.P., Elliott, R.L. and Head, J.F. (2015) Exogenous Normal Mammary Epithelial Mitochondria Suppress Glycolytic Metabolism and Glucose Uptake of $\mathrm{Hu}-$ man Breast Cancer Cells. Breast Cancer Research and Treatment, 153, 519-529. https://doi.org/10.1007/s10549-015-3583-0

[47] Masuzawa, A., Black, K.M., Pacak, C.P., Ericsson, M., Barnett, R.J., Drumm, C., Seth, P., Bloch, D.B., Sevitsky, S., Cowan, D.B. and McCully, J.D. (2013) Transplantation of Autologously Derived Mitochondrial Protects the Heart form Ischemia-Reperfusion Injury. American Journal of Physiology-Heart and Circulatory Physiology, 304, H966-H982. https://doi.org/10.1152/ajpheart.00883.2012

[48] Cowan, D.B., Yao, R., Akurathi, V., Snay, E.R., Thedsanamoorthy, J.K., David Zurakowski, D., et al. (2016) Intracoronary Delivery of Mitochondria to the Ischemic Heart for Cardioprotection. PLoS ONE, 11, e0160889.

https://doi.org/10.1371/journal.pone.0160889

[49] Moskowitzova, K., Orfany, A., Liu, K., Ramirez-Barbieri, G., Thedsanamoorthy, J.K., Yao, R., et al. (2020) Mitochondrial Transplantation Enhances Murine Lung Viability and Recovery after Ischemia-Reperfusion Injury. The American Journal of Physiology_Lung Cellular and Molecular Physiology, 318, L78-L88. https://doi.org/10.1152/ajplung.00221.2019

[50] Hwang, J.W., Lee, M.J., Chung, T.N., Lee, H.A.R., Lee, J.H., Choi, S.Y., et al. (2021) The Immune Modulatory Effects of Mitochondrial Transplantation on Cecal Slurry Model in Rat. Critical Care, 25, 20. https://doi.org/10.1186/s13054-020-03436-X 